\title{
MORSE INEQUALITIES ON CERTAIN INFINITE 2-COMPLEXES
}

\author{
R. AYALA, L. M. FERNÁNDEZ and J. A. VILCHES \\ Dpto. de Geometría y Topología, Universidad de Sevilla, 41080, Sevilla, Spain \\ e-mails:rdayala@us.es,rmfer@us.es,vilches@us.es
}

(Received 22 September, 2005; revised 21 January, 2007; accepted 10 March, 2007)

\begin{abstract}
Using the notion of discrete Morse function introduced by $\mathrm{R}$. Forman for finite $c w$-complexes, we generalize it to the infinite 2-dimensional case in order to get the corresponding version of the well-known discrete Morse inequalities on a non-compact triangulated 2-manifold without boundary and with finite homology. We also extend them for the more general case of a non-compact triangulated 2-pseudo-manifold with a finite number of critical simplices and finite homology.
\end{abstract}

2000 Mathematics Subject Classification. 57M20, 57Q15, 68R10.

1. Introduction. Morse theory is the study of the relationships between functions defined on a space and the shape of this space. More precisely, these relations can be settled by obtaining information of the shape of the space from the information about the critical points of the function. Therefore, the main goal of this theory lies in how the critical points of a function defined on a space affect the topological shape of such space and, conversely, how the shape of a space controls the distribution of the critical points of a function. One way of achieving this goal consists of setting equalities and inequalities between Betti numbers of a given manifold and the numbers of critical points of a Morse function defined on it, the so called Morse inequalities.

R. Forman $[4,5]$ introduced the notion of discrete Morse function defined on a finite $c w$-complex and, in this combinatorial context, he developed a discrete Morse theory as a purely combinatorial tool for studying the topology of the considered complex by means of its homotopy type or homology, for example. In this sense, he proved the corresponding Morse inequalities, analogous to the classical ones obtained in the smooth case. One important application of this result is to define, in a computational way, optimal discrete Morse functions on finite surfaces [6]. In this sense, not only does this discrete Morse theory allow us to check how certain topological theorems are easily accessible via this combinatorial tool, but also the study of the critical points of Morse functions in the context of Combinatorial Differential Topology will provide for us the linkage between discrete gradient fields and the topology of the domain.

The authors are partially supported by the Plan Nacional de Investigación 2.004, Project MTM2004-01865, España, 2005. 
The authors have got a generalized version of discrete Morse inequalities for infinite 1-complexes [1] and this paper is its natural continuation focused on establishing the extension of these inequalities to the infinite 2-dimensional case. As we could expect, for the development of discrete Morse theory on infinite complexes it is necessary to introduce a certain notion of critical element in the infinite, which in some sense will be given by the number of decreasing rays.

Such extension of the discrete Morse inequalities to the non-compact surfaces is interesting for two reasons. First, it reflects homological aspects of such surfaces related to its ends, expressed in terms of its combinatorial structure, but not explicitly linked to its compactification. On the other hand, the obtained inequalities are a non-trivial generalization of the corresponding ones in the finite case because the behaviour of the discrete Morse functions in the infinite is taken into account.

We begin presenting in Section 2 the basic notions concerning infinite discrete Morse theory for later use, following the definitions introduced by R. Forman [4], namely discrete Morse functions, critical simplices and Morse inequalities for the finite case. Moreover, we include in this section some examples which show that Morse inequalities for the finite case do not hold for the infinite case and that some new situations arise when we consider infinite complexes. Section 3 is devoted to the study of discrete Morse functions on infinite 2-complexes. In particular, we introduce the notion of decreasing $i$-rays $(i=1,2)$ which, as we have remarked above, shall play a central role in the main result of this paper. Finally, we shall prove the main result of this paper, Morse inequalities for certain infinite 2-complexes, namely for non-compact triangulated 2-manifolds (surfaces) without boundary and with finite homology, and we extend it to the case of infinite 2-pseudo-manifolds with a finite number of singularities.

2. Preliminaries. As we have announced, we are going to present the notion of discrete Morse function defined on an infinite simplicial complex. It was introduced for the finite case by R. Forman in $[\mathbf{3}, \mathbf{4}, \mathbf{5}]$. Essentially, we shall use the same definition but, since the usual maps between infinite complexes are the proper maps, we shall deal with proper discrete Morse functions, that is, those verifying $f^{-1}([a, b])$ is a finite set for any $a, b \in \mathbf{R}, a \leq b$.

Through all this paper, we consider infinite simplicial complexes which are locally finite. For terminology and background concerning these objects, we refer to [7]. However, for later use we recall that given such a simplicial complex $M$ and a simplex $\sigma \in M$, the star of $\sigma$ in $M$ is defined as the following subcomplex:

$$
\operatorname{st}(\sigma ; M)=\{\tau \in M / \exists \rho \in M \text { with } \tau, \sigma \leq \rho\} .
$$

Moreover, the subcomplex of $\operatorname{st}(\sigma ; M)$ given by

$$
l k(\sigma ; M)=\{\rho \in \operatorname{st}(\sigma ; M) / \sigma \cap \rho=\emptyset\}
$$

is called the link of $\sigma$ in $M$.

On the other hand, an end of $M$ is an equivalence class $[K, C]$ of pairs $(K, C)$ where $K \subset M$ is compact, $C$ is a component of $M-K$ whose closure is not compact and $\left[K_{1}, C_{1}\right]=\left[K_{2}, C_{2}\right]$ if there exists $(K, C)$ with $K_{1} \cup K_{2} \subset K$ and $C \subset C_{1} \cup C_{2}$. 
A discrete Morse function defined on $M$ is a proper function $f: M \longrightarrow \mathbf{R}$ such that, for any $p$-simplex $\sigma^{(p)} \in M$ :

(M1) $\operatorname{card}\left\{\tau^{(p+1)}>\sigma / f(\tau) \leq f(\sigma)\right\} \leq 1$.

(M2) $\operatorname{card}\left\{v^{(p-1)}<\sigma / f(v) \geq f(\sigma)\right\} \leq 1$.

A $p$-simplex $\sigma \in M$ is said to be critical with respect to $f$ if:

(C1) $\operatorname{card}\left\{\tau^{(p+1)}>\sigma / f(\tau) \leq f(\sigma)\right\}=0$.

(C2) $\operatorname{card}\left\{v^{(p-1)}<\sigma / f(v) \geq f(\sigma)\right\}=0$.

From the above definitions, it can be deduced that $\sigma^{(p)}$ is a non-critical simplex if and only if it satisfies one of the following conditions:

(NC1) There exists a simplex $\tau^{(p+1)}>\sigma^{(p)}$ such that $f\left(\tau^{(p+1)}\right) \leq f\left(\sigma^{(p)}\right)$.

(NC2) There exists a simplex $v^{(p-1)}<\sigma^{(p)}$ such that $f\left(v^{(p-1)}\right) \geq f\left(\sigma^{(p)}\right)$.

It is important to point out that both conditions cannot be satisfied simultaneously by a non-critical simplex.

Pictorially, we shall express these two situations as follows:

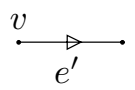

means $f(v) \geq f\left(e^{\prime}\right)$ and we say that $v$ and $e^{\prime}$ are matched; on the other hand,

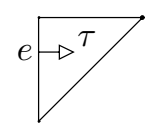

means $f(e) \geq f(t)$ and we say that $e$ and $t$ are matched.

In the finite context, Forman proved the Morse inequalities for discrete Morse functions defined on finite $c w$-complexes [4].

THEOREM 2.1. Let $f$ be a discrete Morse function defined on a finite cw-complex $M$ and let $b_{p}$ be the $p$-th Betti number of $M$ with $p=0, \ldots, \operatorname{dim}(M)$. Then

(I1) $m_{p}(f)-m_{p-1}(f)+\cdots \pm m_{0}(f) \geq b_{p}-b_{p-1}+\cdots \pm b_{0}$,

(I2) $m_{p}(f) \geq b_{p}$

(I3) $m_{0}(f)-m_{1}(f)+m_{2}(f)-\cdots \pm m_{\operatorname{dim}(M)}(f)=b_{0}-b_{1}+b_{2}-\cdots \pm b_{\operatorname{dim}(M)}$, where $m_{p}(f)$ denotes the number of critical p-simplices of $f$ on $M$.

There are examples in which Morse inequalities for the finite case do not hold on an infinite 2-complex (see [1] for examples concerning infinite 1-complexes). For instance, from (I2) of Theorem 2.1 with $p=0$, we get that every finite complex has at least one critical simplex, more precisely, one critical 0 -simplex. However this situation can change when we consider an infinite 2-complex. In this case we can define discrete Morse functions on some non-compact surfaces which do not have any critical simplex. 
Let us consider the following triangulation of the plane $\mathbf{R}^{2}$ and let us define the indicated discrete Morse function on it as shown.

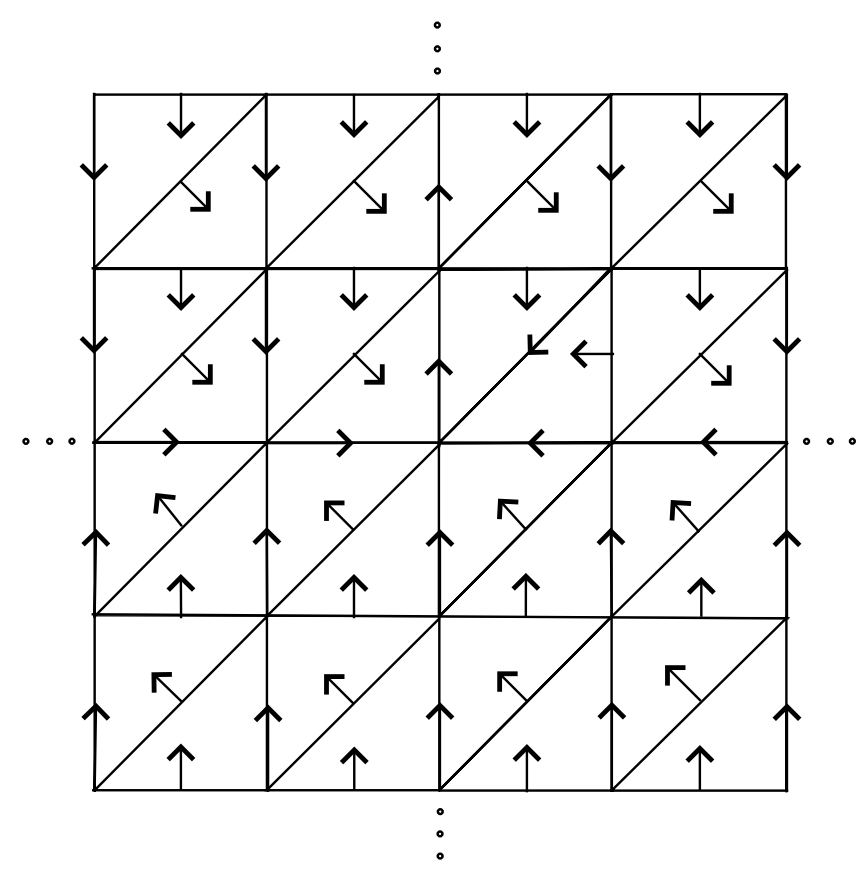

It can be easily checked that there is not any critical simplex for this function on this surface.

These examples justify the necessity of obtaining extended versions of Morse inequalities for infinite complexes. We did this work for the 1-dimensional case [1] and now we are going to carry it out for non-compact 2-manifolds without boundary and with $b_{1}<\infty$.

3. Generalized Morse inequalities on infinite pseudo surfaces. First, we need some general results about discrete Morse functions on infinite complexes. Given an infinite simplicial complex $M$, it is said that a (either finite or infinite) sequence of simplices of $M$,

$$
\alpha_{0}^{(i-1)}, \beta_{0}^{(i)}, \alpha_{1}^{(i-1)}, \beta_{1}^{(i)}, \ldots, \beta_{r}^{(i)}, \alpha_{r+1}^{(i-1)}, \ldots
$$

is a $i$-path $(i=1,2)$ if the $(i-1)$-simplices $\alpha_{n-1}^{(i-1)}$ and $\alpha_{n}^{(i-1)}$ are faces of the $i$-simplex $\beta_{n-1}^{(i)}$, for any $n \in \mathbf{N}$. From now on, we always give the name $i$-ray to any infinite $i$-path. Then, given two $i$-rays contained in the same complex, we say they are equivalent or cofinal if they coincide from a common $(i-1)$-simplex. 
Now, given a discrete Morse function defined on $M$, we say that an $i$-path (resp., $i$-ray),

$$
\alpha_{0}^{(i-1)}, \beta_{0}^{(i)}, \alpha_{1}^{(i-1)}, \beta_{1}^{(i)}, \ldots, \beta_{r}^{(i)}, \alpha_{r+1}^{(i-1)}(, \ldots)
$$

is a decreasing $i$-path (resp., $i$-ray) if

$$
f\left(\alpha_{0}^{(i-1)}\right) \geq f\left(\beta_{0}^{(i)}\right)>f\left(\alpha_{1}^{(i-1)}\right) \geq f\left(\beta_{1}^{(i)}\right)>\cdots \geq f\left(\beta_{r}^{(i)}\right)>f\left(\alpha_{r+1}^{(i-1)}\right)(\geq \cdots) .
$$

Coming back to the last example of Section 2, we can point out that there is a unique decreasing 1-ray and there is not any decreasing 2-ray. As we shall see later, decreasing $i$-rays are closely related to the notion of critical simplices. In fact, roughly speaking, we can say that decreasing rays play the role of critical simplices at the infinite.

It is convenient to point out that, when we consider 2-dimensional infinite simplicial complexes, which are the subjects of this paper, a finite decreasing 1-path always ends in a critical 0 -simplex, since, from the definition of critical 0 -simplex, this is the only one which is not matched with any 1-simplex and hence, it has to be a local minimum of $f$. However, the situation is more complicated for 2-paths. In fact, a decreasing 2-path can either end in a critical 1-simplex, by reasoning in a similar way, or can stop with no relationship with critical 1-simplices. This second option is possible when the decreasing 2-path stops because its last 2-simplex is matched with one of its bordering 1-simplices and its other two bordering 1-simplices are in the same decreasing 1-path or 1-ray. Consequently, there are not cofinal decreasing 2-rays.

We now have the two following lemmas concerning critical simplices of a discrete Morse function and its restrictions. They can be proved in a straightforward way from the definitions of discrete Morse function and critical simplex.

LeMMA 3.1. Let $M$ and $N$ be simplicial complexes such that $N \subseteq M$ and let $f$ be $a$ discrete Morse function defined on $M$. Then the restriction off to $N$ is a discrete Morse function. Moreover, every critical simplex off on $M$ is a critical simplex of the restriction off to $N$ too.

LEMMA 3.2. Let $M$ a 2-complex, $N \subseteq M$ be a subcomplex of $M$ and $f$ be a discrete Morse function defined on $M$. Then any critical 2-simplex of the restriction off to $N$ is a critical 2-simplex off on $M$.

It is interesting to note that Lemma 3.2 does not state anything about simplices of dimensions 0 and 1 . In fact, the number of critical 0 -simplices or 1 -simplices of the restriction of $f$ to $N$ is greater or equal to the number of critical 0 -simplices or 1-simplices of $f$ on $M$.

We are now going to state and prove the main result of this paper: discrete Morse inequalities on a non-compact triangulated surface without boundary and with finite homology. To this end, we shall consider first the connected case. Thus, let $M$ be a noncompact connected triangulated 2-manifold without boundary. So, denoting as above the Betti numbers of $M$ by $b_{i}, i=0,1,2$, we have that $b_{0}=1$ and $b_{2}=0$. Moreover, we suppose that $b_{1}<+\infty$. With these conditions, we deduce that the number of ends of $M$ is finite too. Notice that, since $M$ is a 2-manifold, it admits a locally finite triangulation. 
On the other hand, let $f$ be a (proper) discrete Morse function with a finite number of critical simplices defined on $M$ which induces a finite number of decreasing $i$-rays, $i=1,2$, on $M$.

Next, we consider a connected finite subcomplex of $M, \widehat{M}$, that contains all critical simplices and all basic cycles (which generate the homology) of $M$. Furthermore, since the number of decreasing $i$-rays in $M$ by $f$ is finite, it follows that the number of bifurcations of decreasing 2-rays is finite too, so we can select $\widehat{M}$ verifying that there is not any bifurcation of a decreasing 2-ray in $M-\widehat{M}$. Consequently, since $M$ has a finite number of ends, we can express it as the union of $\widehat{M}$ and a finite number of infinite cylinders, that is,

$$
M=\widehat{M} \cup \bigcup_{j=1}^{n} C_{j},
$$

where $C_{j}$ denotes an infinite cylinder. It is convenient to point out that $C_{j}=K_{j} \times[0, \infty)$ where $K_{j}$ is a 1 -cycle and hence, $C_{j} \cap C_{k}=\emptyset$, for all $j \neq k$. Moreover, since $M$ has a finite number of ends, the boundary of $\widehat{M}$, denoted by $\partial \widehat{M}$, is a finite union of triangulations of $S^{1}$ or, equivalently, a finite union of 1 -cycles, each of them being the intersection of $\widehat{M}$ with one of the cylinders $C_{j}$.

Using Lemmas 3.1 and 3.2, we have that $\widehat{m}_{0} \geq m_{0}, \widehat{m}_{1} \geq m_{1}$ and $\widehat{m}_{2}=m_{2}$, where $m_{i}$ (resp., $\widehat{m}_{i}$ ) denotes the number of critical $i$-simplices of $f$ on $M$ (resp., of the restriction of $f$ to $\widehat{M}), i=0,1,2$. So there can be some critical $i$-simplices of the restriction of $f$ to $\widehat{M}$ which are not critical $i$-simplices of $f$ on $M, i=0,1$. These two possibilities can be found in the example 3.4 .

We are now going to study these particularly interesting situations. Actually, we have that a critical $i$-simplex of the restriction of $f$ to $\widehat{M}$ which is not critical of $f$ on $M$ is either the starting $i$-simplex of a unique decreasing $(i+1)$-path (possibly a decreasing $(i+1)$-ray) contained in $M-\widehat{M}$ or it is the starting $i$-simplex of a decreasing $(i+1)$-path which has a (finite) number of simplices contained in $M-\widehat{M}$ and finally returns to $\widehat{M}$. Note that, in both cases, the starting $i$-simplex has to be in the boundary of $\widehat{M}$, by using the definition of critical simplex we now argue more precisely.

Let $v_{0}$ be a critical 0 -simplex (vertex) of the restriction of $f$ to $\widehat{M}$ which is not a critical 0-simplex of $f$ on $M$. Then, from the definition of discrete Morse function, there exists a unique 1 -simplex (edge) $e_{0}$ in $M-\widehat{M}$ containing $v_{0}$ and such that $f\left(v_{0}\right) \geq f\left(e_{0}\right)$. Let $v_{1}$ be the other vertex of $e_{0}$. It can be either in $\partial \widehat{M}$ or in $M-\widehat{M}$. In the first case, the decreasing 1-path $\left\{v_{0}, e_{0}, v_{1}\right\}$ linked to $v_{0}$ comes back to $\widehat{M}$ at $v_{1}$. However, this decreasing 1-path continues in $M-\widehat{M}$ in the second case and so, since there is no critical simplex of $f$ on $M$ contained in $M-\widehat{M}$, there exists a unique edge $e_{1}$ containing $v_{1}$ in $M-\widehat{M}$ such that $f\left(v_{1}\right) \geq f\left(e_{1}\right)$. Let $v_{2}$ be the other vertex of $e_{1}$. We have two cases again: $v_{2}$ is either in $\partial \widehat{M}$ (and hence we obtain a decreasing 1-path linked to $v_{0}$ which returns to $\widehat{M}$ ) or in $M-\widehat{M}$ and we can continue the process. If the decreasing 1-path linked to $v_{0}$ so constructed never comes back to $\widehat{M}$, then it has to be a decreasing 1 -ray, because it cannot end in a vertex of $M-\widehat{M}$ since, as we have pointed out above, the final 0 -simplex of this decreasing 1-path is a critical vertex and, from the construction of $\widehat{M}$, we know that there are no critical simplices in $M-\widehat{M}$.

A similar process can be described when we consider a critical edge of the restriction of $f$ to $\widehat{M}$ which is not a critical edge of $f$ on $M$. As above, this edge is in $\partial \widehat{M}$ and it 
is the starting 1-simplex of a unique decreasing 2-path. The only difference is that if this 2-path does not come back to $\widehat{M}$, that is, if it is totally contained in $M-\widehat{M}$, then either it is a 2 -ray or it can end in a 2 -simplex of $M-\widehat{M}$.

Observe that, since $\partial \widehat{M}$ is a finite union of finite 1-cycles, there can be only a finite number of critical $i$-simplices $(i=0,1)$ of the restriction of $f$ to $\widehat{M}$ which are not critical simplices of $f$ on $M$.

Next, under the stated conditions and notations concerning $M, f$ and $\widehat{M}$, we have the following lemma.

LEMMA 3.3. There exists a connected finite subcomplex $\bar{M}$ of $M$ containing all critical simplices off on $M$, all basic cycles of $M$, such that there are not bifurcations of decreasing 2-rays in $M-\bar{M}$ and such that if there is a critical $i$-simplex of the restriction off to $\bar{M}$ which is not a critical $i$-simplex off on $M$, then it is linked to a unique decreasing $(i+1)$-ray totally contained in $M-\bar{M}$, for $i=0,1$.

Proof. In order to reduce the terminology, given a subcomplex $N$ of $M$, we call any non-critical $i$-simplex of $f$ on $M$ distinguished with respect to $N$, if:

(i) it is a critical $i$-simplex of $f$ on $N$;

(ii) it is not linked to a decreasing $(i+1)$-ray.

Then the objective of the lemma is to find a finite subcomplex of $M$ containing all critical simplices of $f$ on $M$, all basic cycles of $M$, verifying that there are not bifurcations of decreasing 2-rays in $M-\bar{M}$ and such that it does not contain distinguished $i$-simplices, $i=0,1$.

First we consider $\widehat{M}$, constructed as above. If all the critical $i$-simplices $(i=0,1)$ of the restriction of $f$ to $\widehat{M}$ which are not critical $i$-simplices of $f$ on $M$ (we know that they are in in $\partial \widehat{M})$ are linked to decreasing $(i+1)$-rays, then we take $\bar{M}=\widehat{M}$. Otherwise, we are going to construct $\bar{M}$ from $\widehat{M}$ by using the following procedure. Let $v_{0}$ be a distinguished 0 -simplex with respect to $\widehat{M}$, if there is any. Then $v_{0}$ is the starting 0 -simplex of a unique finite decreasing 1-path contained in $M-\widehat{M}$ which comes back to $\widehat{M}$. Thus, let $v_{1}$ be the last vertex of this decreasing 1-path. It is clear that $v_{1}$ is in $\partial \widehat{M}$. So, we consider the 1 -cycle made up by the union of such decreasing 1-path with the 1-path contained in $\partial \widehat{M}$ which joins $v_{0}$ and $v_{1}$ and such that such a 1-cycle is the boundary of a triangulated closed disk. We add to $\widehat{M}$ this disk and we denote this new subcomplex by $\widehat{M}_{v_{0}}$. Observe that, now, $v_{0}$ is not a distinguished vertex with respect to $\widehat{M}_{v_{0}}$. Moreover, this procedure does not introduce new distinguished simplices with respect to $\widehat{M}_{v_{0}}$, since the part of the boundary of the added triangulated disk contained in $\partial \widehat{M}_{v_{0}}$ is a decreasing 1-path and, hence, all its simplices are matched, in particular $v_{1}$.

We repeat this process with all the remaining distinguished vertices with respect to $\widehat{M}_{v_{0}}$ (a finite number, in any case) and we get a finite subcomplex $\widehat{M}_{v}$ in which there is no distinguished 0 -simplex.

Now let $e_{0}$ be a distinguished 1-simplex with respect to $\widehat{M}_{v}$, if there is any. Then $e_{0}$ is the starting edge of a unique decreasing finite 2-path which either comes back to $\widehat{M}_{v}$ or is totally contained in $M-\widehat{M}_{v}$.

In the first case, we construct a new subcomplex, denoted by $\widehat{M}_{e_{0}}$, by adding to $\widehat{M}_{v}$ such decreasing 2-path (including all the boundary 1-simplices of its 2-simplices) and the finite subcomplex bounded by that 2-path and $\partial \widehat{M}_{v}$. Then, $e_{0}$ is not a distinguished edge with respect to $\widehat{M}_{e_{0}}$. Moreover, none of the added simplices is distinguished with respect to $\widehat{M}_{e_{0}}$ because the boundary of any decreasing 2-path in $M-\widehat{M}_{v}$ is made up by decreasing 1-paths, and hence all the new simplices are matched. 
On the other hand, if $e_{0}$ is the starting 1-simplex of a decreasing finite 2-path totally contained in $M-\widehat{M}_{v}$, we construct $\widehat{M}_{e_{0}}$ in a similar way by adding this 2 path (including all its boundary 1 -simplices) to $\widehat{M}$. Again, $e_{0}$ is not distinguished with respect to $\widehat{M}_{e_{0}}$. Moreover, by using the same argument as above, all the added simplices cannot be distinguished with respect to $\widehat{M}_{e_{0}}$.

We repeat this process with all the remaining distinguished edges with respect to $\widehat{M}_{e_{0}}$ (a finite number, in any case) and, finally, we get a finite subcomplex $\bar{M}$ in which there is not any distinguished simplex.

In order to clarify the process described in the above lemma, we are going to work it out in the following examples:

EXAMPLE 3.4. Let us consider the following triangulation of an infinite cylinder $C_{j}$, in which we mark $\partial \widehat{M}$ by dotted lines and decreasing 1-paths by thicker lines.

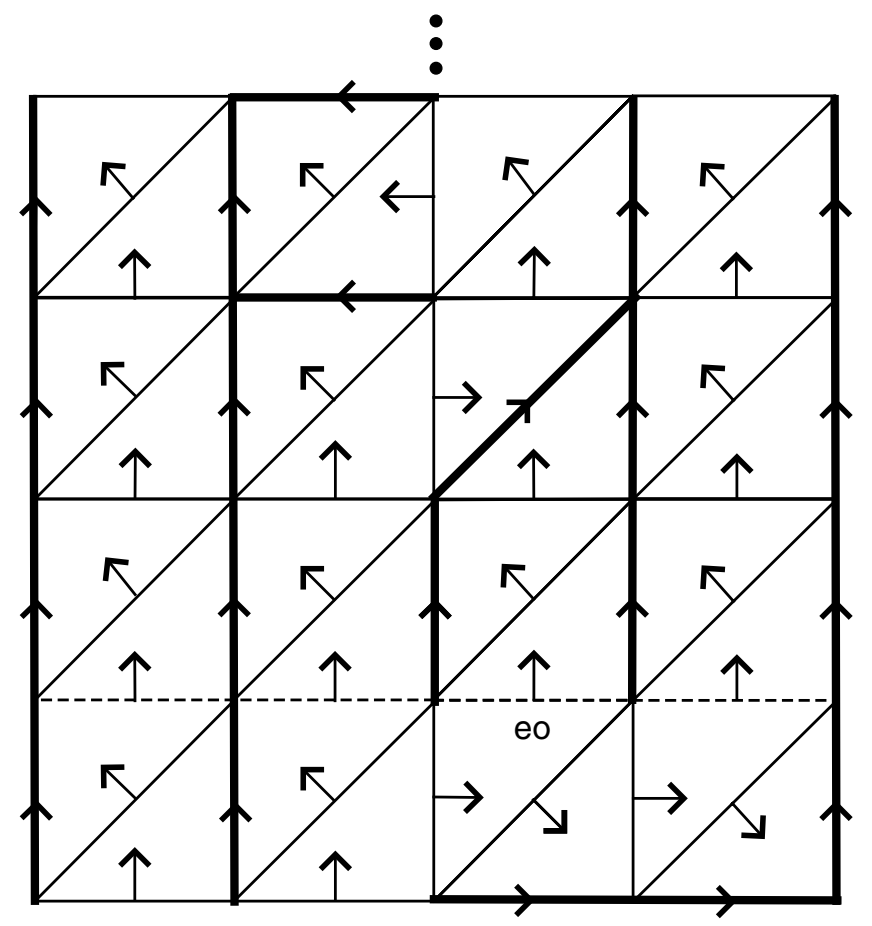

It is convenient to point out that in this example there is not any distinguished vertex with respect to $\widehat{M}$. Notice that $e_{0}$ is the starting edge of a decreasing 2-path totally contained in $M-\widehat{M}$ and hence it is a distinguished edge with respect to $\widehat{M}$. So we add this 2-path to $\widehat{M}$ to get a new subcomplex $\bar{M}$ in which the edge $e_{0}$ is not distinguished.

EXAMPLE 3.5. Again, let us consider a triangulation of an infinite cylinder $C_{j}$, in which we mark $\partial \widehat{M}$ by dotted lines and decreasing 1-paths by thicker lines.

Since $v_{0}$ is the starting vertex of a decreasing 1-path which comes back to $\widehat{M}$ at $v_{1}$, $v_{0}$ is a distinguished vertex with respect to $\widehat{M}$. By the procedure described in the proof of Lemma 3.3, we add to $\widehat{M}$ this finite decreasing 1-path and all simplices contained in the subcomplex bounded by this 1-path and the edge $e_{0}$. So we get a new finite 
subcomplex $\bar{M}$ in which $v_{0}$ is not distinguished with respect to $\bar{M}$. Notice that in this construction, the edge $e_{0}$, which is distinguished with respect to $\widehat{M}$, is replaced by the 1-path joining $v_{0}$ and $v_{1}$, and hence, is not a distinguished edge with respect to $\bar{M}$.

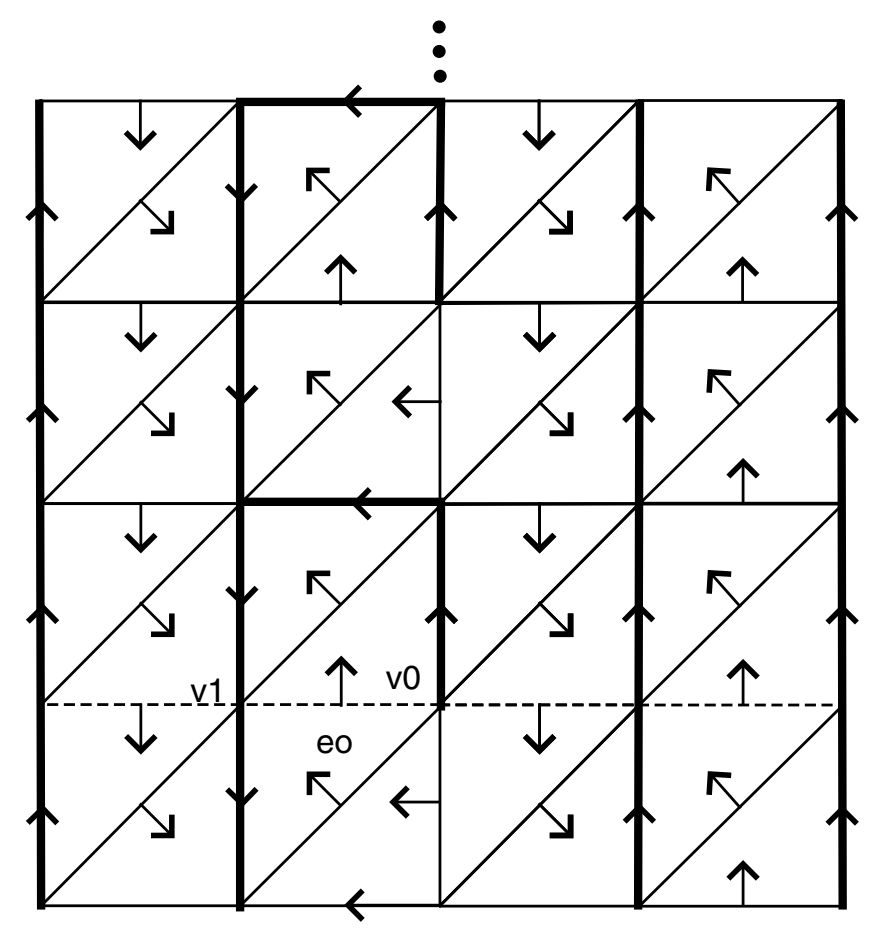

At this point we can prove the main result of this paper.

THEOREM 3.6. Let $M$ be a non-compact connected triangulated 2-manifold without boundary such that $b_{1}<+\infty$. Let $f$ be a discrete Morse function defined on $M$ with a finite number of critical simplices and a finite number of decreasing $i$-rays, $i=1,2$. Then

(1) $m_{0}+d_{0} \geq 1 ; m_{1}+d_{1} \geq b_{1}$;

(2) $m_{1}+d_{1}-m_{0}-d_{0} \geq b_{1}-1 ; m_{2}-m_{1}-d_{1}+m_{0}+d_{0} \geq 1-b_{1}$;

(3) $m_{0}+d_{0}-m_{1}-d_{1}+m_{2}=1-b_{1}$,

where $d_{i}$ denotes the number of non equivalent decreasing $(i+1)$-rays off in $M, i=0,1$.

Proof. We consider a finite subcomplex $\bar{M}$ of $M$ as described in Lemma 3.3. Then every critical $i$-simplex of the restriction of $f$ to $\bar{M}$ which is not critical of $f$ on $M$ is linked to a decreasing $(i+1)$-ray, for $i=0,1$ and all of them are contained in $\partial \bar{M}$.

If there exist two different vertices in $\partial \bar{M}$, say $v_{0}$ and $v_{1}$ which are linked to two decreasing 1-rays which converge in $M-\bar{M}$, that is, there exists another vertex $v_{2}$ in $M-\bar{M}$ such that it is linked to a unique decreasing 1-ray and the 1-path from $v_{0}$ (resp., from $v_{1}$ ) to $v_{2}$ is a decreasing one. So these two 1-rays are cofinal. Then, we proceed in a way similar to that used in Lemma 3.3 and we add to $\bar{M}$ the finite subcomplex bounded by both decreasing 1-paths and the 1-path in $\partial \bar{M}$ joining $v_{0}$ and $v_{1}$ (including these boundary 1-paths). Note that this process is finite due to the fact that the original $\partial \bar{M}$ is finite and that the "new" $\bar{M}$ has the same properties as the "old" one. 
Consequently, we have a one to one map between the set of critical $i$-simplices of the restriction of $f$ to $\bar{M}$ which are not critical of $f$ on $M$ and the set of decreasing $i+1$-rays in $M$. Hence, this map is expressed by the following equality,

$$
m_{i}+d_{i}=\bar{m}_{i}
$$

with $i=0,1$, where $\bar{m}_{i}$ denotes the number of critical $i$-simplices of the restriction of $f$ to $\bar{M}$.

Finally, we get the result by applying Theorem 2.1 to $\bar{M}$ and by using (3.1).

We are going to extend Theorem 3.6 to more general situations. First we suppose that $M$ has $k$ connected components. Then $b_{0}=k$. Repeating the argument on each connected component and taking $\bar{M}$ as the union of the finite subcomplexes obtained in them, we have the following result.

THEOREM 3.7. Let $M$ be a non-compact triangulated 2-manifold without boundary such that $b_{1}<+\infty$. Let $f$ be a discrete Morse function defined on $M$ with a finite number of critical simplices and a finite number of decreasing $i$-rays, $i=1,2$. Then

(1) $m_{0}+d_{0} \geq b_{0} ; m_{1}+d_{1} \geq b_{1}$;

(2) $m_{1}+d_{1}-m_{0}-d_{0} \geq b_{1}-b_{0} ; m_{2}-m_{1}-d_{1}+m_{0}+d_{0} \geq b_{0}-b_{1}$;

(3) $m_{0}+d_{0}-m_{1}-d_{1}+m_{2}=b_{0}-b_{1}$,

where $d_{i}$ denotes the number of non equivalent decreasing $(i+1)$-rays off in $M, i=0,1$.

Finally, we consider the case when $M$ is a non-compact 2-pseudo-manifold with $b_{1}<+\infty$ and with a finite number of singular simplices, that is, $M$ has a finite number of vertices whose link in $M$ is not a 1-cycle and a finite number of edges $e$ verifying that $\operatorname{card}\{l k(e ; M)\}>2$. In this case, $0 \leq b_{2}<+\infty$. If we use a similar argument but take $\bar{M}$ in such a way that, moreover, its Betti number $\bar{b}_{2}$ is equal to $b_{2}$ or, equivalently, that it contains all the homology of $M$, which is possible due to the fact that is is finite, then we can prove the following theorem.

THEOREM 3.8. Let $M$ be a non-compact triangulated 2-pseudo-manifold with a finite number of singular simplices such that $b_{1}<+\infty$. Let $f$ be a discrete Morse function defined on $M$ with a finite number of critical simplices and a finite number of decreasing $i$-rays, $i=1,2$. Then

(1) $m_{0}+d_{0} \geq b_{0} ; m_{1}+d_{1} \geq b_{1} ; m_{2} \geq b_{2} ;$

(2) $m_{1}+d_{1}-m_{0}-d_{0} \geq b_{1}-b_{0} ; m_{2}-m_{1}-d_{1}+m_{0}+d_{0} \geq b_{2}-b_{1}+b_{0}$;

(3) $m_{0}+d_{0}-m_{1}-d_{1}+m_{2}=b_{2}-b_{1}+b_{0}$,

where $d_{i}$ denotes the number of non equivalent decreasing $(i+1)$-rays off in $M, i=0,1$.

Observe that Theorem 3.8 is the natural generalization of Theorem 2.1 to the infinite 2-dimensional case.

ACKNOWLEDGEMENT. We would like to thank to the referee for his valuable comments which led to improvements in this paper.

\section{REFERENCES}

1. R. Ayala, L. M. Fernández and J. A. Vilches, Desigualdades de Morse generalizadas sobre grafos, Actas de las III jornadas de matemática discreta y algorítmica (Universidad de Sevilla, Sevilla, 2002), 159-164. 

1997).

2. R. Diestel, Graph theory, Graduate texts in mathematics, No. 173 (Springer-Verlag,

3. R. Forman, Combinatorial differential topology and geometry, New perspectives in geometric combinatorics (MSRI Publications, No. 38, 1999), 177-206.

4. R. Forman, Morse theory for cell complexes, Adv. Math. 134 (1998), 90-145.

5. R. Forman, Combinatorial vector fields and dynamical systems, Math. Z. 228 (1998), 629-681.

6. T. Lewiner, H. Lopes and G. Tavares, Optimal discrete Morse functions for 2-manifolds, in Computational geometry: theory and applications (Elsevier Science, 2003), 221-233.

7. J. R. Munkres, Elements of algebraic topology (Addison-Wesley, 1984).

8. J. A. Vilches, Funciones de Morse dicretas sobre complejos infinitos, Ph.D. Thesis (Universidad de Sevilla, Sevilla, 2003). 